\title{
A BOUNDS TAUBERIAN THEOREM
}

\author{
AlLen Stenger
}

Abstract. We weaken the hypothesis and the conclusion of a Hardy-Littlewood Tauberian theorem, and apply the new theorem to deduce asymptotic behavior of the coefficients of an exponentiated lacunary series.

Mathematics subject classification (2010): 40E05, 41A60.

Keywords and phrases: Tauberian theorems, asymptotics of sequences, lacunary series.

\section{REFERENCES}

[1] G. H. HaRdy, On certain oscillating series, Q. J. Math. 38 (1907) 269-288. Reprinted with corrections in Collected Papers of G. H. Hardy, volume VI, pp. 146-167; Oxford Univ. Press, Oxford, 1974.

[2] G. H. Hardy, J. E. Littlewood, Tauberian theorems concerning power series and Dirichlet's series whose coefficients are positive, Proc. Lond. Math. Soc. (2) 13 (1914) 174-191. Reprinted with corrections in Collected Papers of G. H. Hardy, volume VI, pp. 510-529; Oxford Univ. Press, Oxford, 1974.

[3] J. KorevaAR, Tauberian Theory: A Century of Developments, Springer, Berlin, 2004.

[4] G. Stoica, Problem proposed: 11849, Amer. Math. Monthly 122 (2015) 605.

[5] G. Stoica, A. Stenger, Asymptotics for Coefficients: 11849, Amer. Math. Monthly 124 (2017) 378.

[6] E. C. Titchmarsh, The Theory of Functions, Second edition, Oxford Univ. Press, Oxford, 1939. 